\title{
The status of PAH gene-VNTR alleles and mini-haplotypes associations with $P A H$ gene mutations in Iranian Kurdish PKU patients
}

\author{
Reza Alibakhshi*1, Keivan Moradi², Keyghobad Ghadiri ${ }^{3}$ \\ Received: 27 Nov 2017 \\ Published: 26 Aug 2019
}

\section{Abstract}

Background: The analysis of haplotypes/mini-haplotypes in the $P A H$ gene has been used as an informative tool in several genetic anthropology studies. Considering the notion that Iranian population is one of the most heterogeneous $i$ the world, this study was conducted to evaluate the association of VNTR-STR mini-haplotypes with the $P A H$ gene mutations in PKU patients in Kermanshah province.

Methods: A total of 24 unrelated Kurdish PKU patients with the known PAH gene causing mutations and 72 healthy controls were selected. The DNA fragments containing VNTR and STR systems were amplified by polymerase chain reaction (PCR). For VNTR system, PCR products were separated using electrophoresis on $2.5 \%$ agarose gel. For STR system, the samples were analyzed using DNA sequencing analysis version 5.2 software.

Results: Overall, $5 P A H$-VNTR-alleles, including VNTR3, 7, 8, 9, 12, and $3 P A H$-STR-alleles, including STR238, 242, and 250, were detected in this study. VNTR3 and 8 alleles had the most frequency among healthy controls. Also, 6 different mini-haplotype alleles were found to be associated with PKU chromosomes. The 2 most prevalent mutations in Kermanshah province, IVS2+5G $>\mathrm{C}$ and IVS $9+5 \mathrm{G}>\mathrm{A}$, were strongly linked to mini-haplotypes $9 / 242$ and $8 / 238$, respectively.

Conclusion: The distributions and frequencies of VNTR alleles in Kurdish population have the most similarity to alleles previously described in European Caucasian families. Moreover, since the most common mutations in Kermanshah PKU chromosomes are rare and this was the first study on mini-haplotypes VNTR/STR among Iranian Kurdish PKU patients, given that this study was the first of its kind, it was not possible to compare its results with that of other studies on Iranian and non-Iranian populations.

Keywords: $P A H$, VNTR, STR, Mini-Haplotype, Iran

Conflicts of Interest: None declared

Funding: Vice Chancellor for Research at Kermanshah University of Medical Sciences

\section{*This work has been published under CC BY-NC-SA 1.0 license.}

Copyright $₫$ Iran University of Medical Sciences

Cite this article as: Alibakhshi R, Moradi K, Ghadiri K. The status of $P A H$ gene-VNTR alleles and mini-haplotypes associations with $P A H$ gene mutations in Iranian Kurdish PKU patients. Med J Islam Repub Iran. 2019 (26 Aug);33:88. https://doi.org/10.47176/mjiri.33.88

\section{Introduction}

Phenylketonuria [PKU; McKusick OMIM 261600], a developmental disorder with general cognitive disabilities (1) and a type of inborn error of phenylalanine metabo-

\section{Corresponding author: Dr Reza Alibakhshi, ralibakhshi3@gmail.com}

1. Department of Biochemistry, School of Medicine, Kermanshah University of Medical Sciences, Kermanshah, Iran

2. Medical Biology Research Center, Kermanshah University of Medical Sciences, Kermanshah, Iran

3. Infectious Disease Research Center, Kermanshah University of Medical Sciences, Kermanshah, Iran lism, is one of the most common of the 300 or so known inherited metabolic diseases (2). Mutations in the Phenylalanine Hydroxylase or $P A H$ gene (NCBI Gene ID: 5053),

$\uparrow$ What is "already known" in this topic:

- Some believe that Iranian population is one of the most heterogeneous populations of the world.

- VNTR-STR mini-haplotypes in the $P A H$ gene are highly informative for mutation analysis, including prenatal diagnosis phenylketonuria.

$\rightarrow$ What this article adds:

- This study evaluated the frequencies of the $P A H$-gene-VNTR alleles for the first time in healthy controls in Kermanshah province and confirmed a high degree of heterogeneity among this population.

- This was the first report of association of VNTR-STR minihaplotypes and $P A H$ gene mutations in Iranian Kurdish PKU patients. 
Table 1. Oligonucleotide amplification primers, annealing temprature, location, and length of PCR products

\begin{tabular}{lcccc}
\hline $\begin{array}{l}\text { Polymorphism } \\
\text { system }\end{array}$ & Primer sequence (5'-3') & $\begin{array}{c}\text { Annealing } \\
\text { temperature }\end{array}$ & Location in PAH gene & Length (bp) \\
\hline VNTR & $\begin{array}{l}\text { (F) GCTTGAAACTTGAAAGTTGC } \\
\text { (R) GGAAACTTAAGAATCCCATC }\end{array}$ & $56^{\circ} \mathrm{C}$ & $\begin{array}{l}3^{\prime} \text { untranslated region } \\
3^{\prime} \text { untranslated region }\end{array}$ & Vary according to 30bp cassettes \\
\hline STR & $\begin{array}{l}\text { (F) GCCAGAACAACTACTGGTTC } \\
\text { (R) AATCATAAGTGTTCCCAGAC }\end{array}$ & $58^{\circ} \mathrm{C}$ & $\begin{array}{l}\text { Intron 3 } \\
\text { Intron 3 }\end{array}$ & Vary according to TCTA repeats \\
\hline
\end{tabular}

located on chromosome 12 in humans, are the main reason for phenylketonuria. This gene contains 13 exons and encodes 452 amino acids (3). According to the $P A H$ locus database (http://www.pahdb.mcgill.ca), more than 800 $P A H$ gene mutations have been identified and recorded (4).

The analysis of a variable number of tandem repeat or VNTR of 30-bp cassettes in the $P A H$ gene has been used as an informative tool in several genetic anthropology studies (5-8). Geographical location and having a high level of linguistic diversity and different ethnicities make Iran one of the most interesting regions to investigate the relationship between ethnic and genetic processes $(9,10)$. Previous studies have found a great genetic diversity among Iranian populations (11). Therefore, this study aimed to examine the $P A H$-VNTR status in Iranian Kurdish population living in Kermanshah province and to compare the results with other studies performed on this subject in Iran and other parts of the world.

Along with a large number of informative haplotypes derived from $P A H$ locus polymorphic markers, including several RFLPs, a VNTR, and a series of short tandem repeats or STRs of tetra-nucleotides TCTA (12-14), a VNTR/STR mini-haplotype was developed (3, 12, 15). Due to a Mendelian fashion inheritance and high polymorphic nature, these last 2 genetic markers could be used to give a risk estimation of linked defective alleles, alone or in combination. Moreover, this VNTR may prove useful in studies on the origins and distributions of $P A H \mathrm{mu}-$ tations in different human populations $(3,12,13)$. According to our previous studies (16-20), there are at least 15 different $P A H$-causing mutations, including 2 novel mutations, possibly specific to Kurdish population, among phenylketonuria patients in Kermanshah province. Therefore, the aim of this study was to check mini-haplotype associations of the most frequent $P A H$ gene mutations in PKU patients in Kermanshah province.

\section{Methods \\ Patients}

This study was conducted over a period of 18 months (2014-2015) at Kermanshah University of Medical Sciences, Medical Genetics Laboratory, Kermanshah, Iran. A total of 24 unrelated PKU patients in Kermanshah province, with Kurdish ethnicity and known $P A H$ genecausing mutations (16) enrolled in the study. The diagnosis of patients had been based on clinical criteria/laboratory findings (detection of elevated Phe levels in blood samples using HPLC) and disease confirmation was done by molecular genetic analysis. After ethical approval of the project by the ethics committee of Kermanshah University of Medical Sciences (the project and ethics committee code \# 89184) and obtaining informed consent forms, the patients and 72 healthy matched controls, with no genetic and congenital diseases, were tested for minihaplotypes (VNTR/STR) in the $P A H$ gene. Rationale for the selection of control to case ratio, 3:1, was based on Kang et al study (21).

\section{Experimental methods}

Genomic DNA extraction was performed following the manufacturer's recommendations of a QIAamp DNA Mini-Kit (Qiagen, USA). Polymerase chain reaction (PCR) amplification was performed in an ABI thermocycler (Applied Biosystems PCR System 9700) using primers shown in Table 1. PCR conditions for amplification of VNTR and STR were as follow: initial denaturation at $95^{\circ} \mathrm{C}$ for 5 minutes; 30 cycles including denaturation at $95^{\circ} \mathrm{C}$ for 60 seconds, annealing at $56^{\circ} \mathrm{C}$ (for VNTR) and $58^{\circ} \mathrm{C}$ (for STR) for 60 seconds, and elongation at $72^{\circ} \mathrm{C}$ for 90 seconds in each cycle; and final elongation at $72^{\circ} \mathrm{C}$ for 5 minutes. For VNTR system, PCR products were separated using electrophoresis on $2.5 \%$ agarose gel. Since the amplified products of VNTR may differ by their lengths, a 50-bp DNA-marker was used.

\section{Sequence analysis}

For STR system, 2\% agarose gel was stained with green-dye for visualizing the fragment migration. Then, a number of 16 samples whose homozygous $P A H$ genecausing mutations were obtained previously and had consanguineous parents (first relatives), which were analyzed by direct sequencing in an ABI-3130 DNA analyzer (Applied Biosystems, USA). PCR products were purified using QIAquick PCR purification kit (Qiagen, USA). After ethanol-sodium acetate precipitation and cycle sequencing of sense and antisense strands, the data were analyzed using DNA sequencing analysis version 5.2 software (Applied Biosystems, USA), and the obtained sequence was compared with the $P A H$ gene original sequence.

\section{Results}

Overall, 5 PAH-VNTR and 3 PAH-STR-alleles were detected in this study. The lengths of amplified VNTR products were $364,484,514,544$, and 634 bp corresponding to the presence of alleles with $3,7,8,9$, and 12 copies of the repeated units, respectively (Table 2). While all 5 alleles were shown in the control group, the VNTR12 allele was absent in the patients. The frequencies of VNTR3, 7, 8, 9, and 12 alleles were as follow: $3(6.25 \%)$, $11(22.92 \%), 19(39.58 \%), 15(31.25 \%)$, and $0(0 \%)$ in patients and $60(41.67 \%), 17(11.80 \%), 46(31.95 \%), 17$ $(11.80 \%)$, and $4(2.78 \%)$ in controls. From 15 possible genotypes derived from combinations of these alleles, 7 and 12 genotypes were observed in the patients and con- 


\begin{tabular}{|c|c|c|c|}
\hline \multicolumn{2}{|c|}{ VNTR-polymorphisms } & Patients $(\mathrm{N}=24)$ & Controls $(\mathrm{N}=72)$ \\
\hline \multirow{5}{*}{ 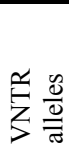 } & VNTR3 & $3(6.25)$ & $60(41.67)$ \\
\hline & VNTR7 & $11(22.92)$ & $17(11.80)$ \\
\hline & VNTR8 & $19(39.58)$ & $46(31.95)$ \\
\hline & VNTR9 & $15(31.25)$ & $17(11.80)$ \\
\hline & VNTR12 & $0(0)$ & $4(2.78)$ \\
\hline \multirow{15}{*}{ 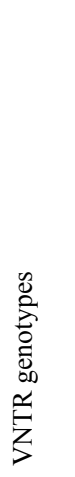 } & VNTR3/VNTR3 & $0(0)$ & $13(18.05)$ \\
\hline & VNTR3/VNTR7 & $0(0)$ & $4(5.55)$ \\
\hline & VNTR3/VNTR8 & $1(4.17)$ & $19(26.39)$ \\
\hline & VNTR3/VNTR9 & $2(8.33)$ & $8(11.11)$ \\
\hline & VNTR3/VNTR12 & $0(0)$ & $3(4.17)$ \\
\hline & VNTR7/VNTR7 & $5(20.83)$ & $3(4.17)$ \\
\hline & VNTR7/VNTR8 & $1(4.17)$ & $7(9.72)$ \\
\hline & VNTR7/VNTR9 & $0(0)$ & $1(1.39)$ \\
\hline & VNTR7/VNTR12 & $0(0)$ & $0(0)$ \\
\hline & VNTR8/VNTR8 & $7(29.17)$ & $8(11.11)$ \\
\hline & VNTR8/VNTR9 & $3(12.50)$ & $4(5.56)$ \\
\hline & VNTR8/VNTR12 & $0(0)$ & $0(0)$ \\
\hline & VNTR9/VNTR9 & $5(20.83)$ & $1(1.39)$ \\
\hline & VNTR9/VNTR12 & $0(0)$ & $1(1.39)$ \\
\hline & VNTR12/VNTR12 & $0(0)$ & $0(0)$ \\
\hline
\end{tabular}

Table 3. The association of $P A H$ gene mutations with VNTR and VNTR/STR mini-haplotype in Kurdish PKU patients. The patients were divided into 2 groups: patients who had a homozygous mutation or heterozygous mutation. For the first group, the VNTR/STR mini-haplotype was investigated while only the VNTR was checked for the second group.

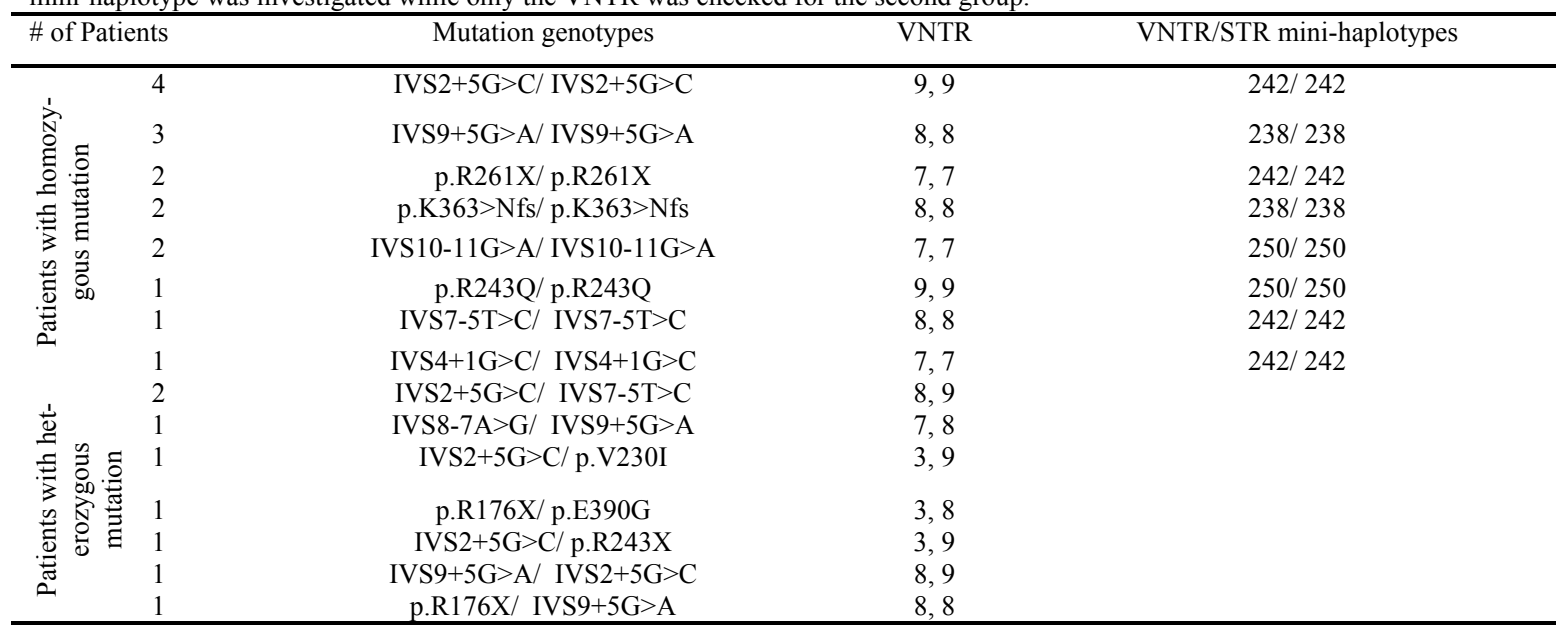

trols, respectively. The genotypes of VNTR8/VNTR8 (29.17\%), VNTR9/VNTR9 (20.83\%), and VNTR7/VNTR7 (20.83\%) in the patients and VNTR3/VNTR8 (26.39\%), VNTR3/VNTR3 (18.05\%), VNTR3/VNTR9 (11.11\%), and VNTR8/VNTR8 $(11.11 \%)$ in the controls had the highest frequencies in this study (Table 2).

After analyzing VNTR alleles, it was found that 7 out of 24 patients were homozygous for VNTR8, 5 for VNTR9, and 5 for VNTR7. Also, 3 mutations (IVS9+5G $>A$, p.K363 $>$ Nfs and IVS7-5 T $>$ C), 2 mutations (IVS2 $+5 \mathrm{G}>\mathrm{C}$ and p.R243Q), and 3 mutations (IVS10-11G $>$ A, p.R261X and IVS4 $+1 \mathrm{G}>\mathrm{C}$ ) were associated with VNTR8, 9, and 7 alleles, respectively (Table 3). Moreover, 6 different VNTR/STR mini-haplotypes, including 9:242, 8:238, $7: 242,7: 250,9: 250$, and $8: 242$, were found to be associated with PKU chromosomes in patients with homozygous mutation (Table 3). A segment of $P A H$ - gene-STR sequence chromatogram with a periodic pattern of TCTA is shown in Figure 1. This segment was common among 3 types of STRs identified in this study $(238,242$, and 250).
According to the results of this study, the 2 most prevalent $P A H$ gene mutations among PKU patients in Kermanshah province (16), IVS2 $+5 \mathrm{G}>\mathrm{C}$ and IVS9 $+5 \mathrm{G}>\mathrm{A}$, were completely linked to mini-haplotypes $9 / 242$ and $8 / 238$, respectively. IVS $4+1 \mathrm{G}>\mathrm{C} \quad($ c. $441+1 \mathrm{G}>\mathrm{C})$ and IVS7-5T $>C$ (c.843-5T $>C)$ are 2 novel mutations that may be specific to Kurdish population. Results of this study demonstrated that these 2 mutations are linked to mini-haplotypes $7 / 242$ and $8 / 242$, respectively (16).

\section{Discussion}

According to Da Silva et al (7) and ALFRED database (https://alfred.med.yale.edu/alfred/index.asp), there are at least 12 PAH-VNTR alleles in the human genome. Several studies have been conducted in Iran $(5,22-28)$ and other parts of the world (15) which reported PAH-VNTR alleles in PKU patients and healthy controls; however, their types and frequencies varied among different populations.

Overall, 5 VNTR alleles and 12 VNTR genotypes were detected in 24 PKU patients and 72 matched healthy controls in Kermanshah province, Iran. VNTR3 and VNTR8 


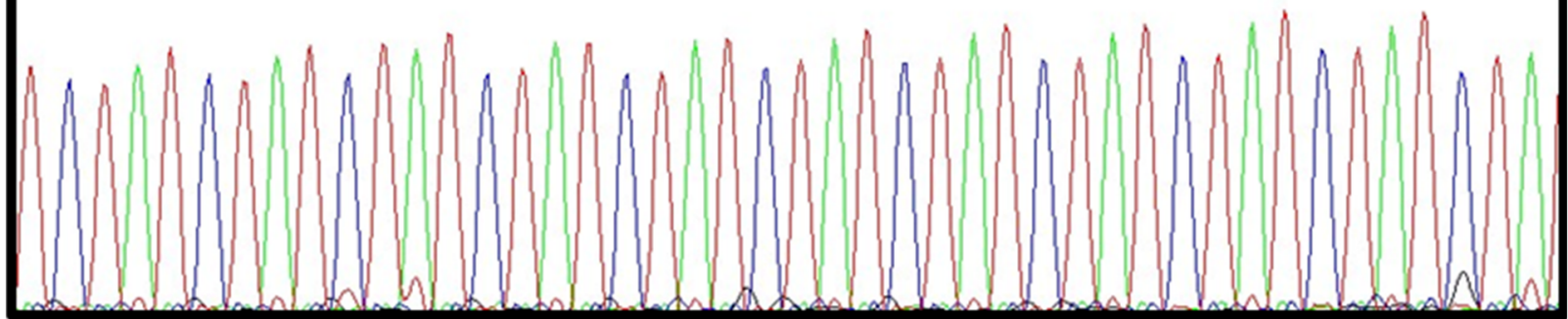

Fig. 1. Example of a periodic pattern of TCTA in $P A H$-gene-STR system. This segment was common among three types of STRs identified in this study $(238,242$ and 250$)$.

were the 2 most frequent alleles in our normal samples with the frequencies of $41.67 \%$ and $31.3 \%$, respectively. Also, VNTR3/VNTR8 and VNTR3/VNTR3 genotypes had the most frequencies among genotypes detected. The results of this study are similar to those of the studies conducted by Hosseini-Mazinani et al, who performed a comprehensive study on this subject with samples from all parts of Iran (5) and Kamkar et al in Fars province (24). However, the results of the present study significantly differed with those of studies performed in West Azerbaijan (23), Isfahan (25), and Yazd (22) provinces (Table 4). Overall, the results of this study and other studies conducted in Iran are compatible with the idea that Iranian population is one of the most heterogeneous populations of the world $(29,30)$. Moreover, Derenko et al (11) proved an extremely high level of genetic diversity in the Iranian population based on the complete mtDNA sequence analysis, which is comparable to the other groups from the South Caucasus, Anatolia, and Europe. Due the high similarity between the distribution and frequency of VNTR alleles in Kurdish population and European Cau- casian families (6), it seems that these results are compatible with Derenko et al study (11) (Table 4).

According to our previous studies (16-20), there are at least 15 different $P A H$ gene-causing mutations among PKU patients in Kermanshah province. To our knowledge, this was one of the rare studies on mini-haplotypes VNTR/STR among PKU patients from Iran.

Unfortunately, the data on mini-haplotypes were linked to IVS2+5G $>$ C and IVS9+5G $>$ A, as the most frequent mutations in individuals with PKU in Kermanshah province, are rare in the literature (Table 5). In the present study, IVS $2+5 \mathrm{G}>\mathrm{C}$ and IVS9 $+5 \mathrm{G}>\mathrm{A}$ mutations were strongly associated with mini-haplotype 9/242 and 8/238, respectively that are compatible with the results of Razipour et al. (12). The IVS10-11G $>$ A is a common mutation in parts of Southern Europe and in Mediterranean countries and Iran (20). This mutation has been found on several distinct mini-haplotypes, most commonly on mini-haplotype $7 / 250(15,27)$ (Table 5). All of 4 PKU chromosomes harboring mutation IVS10-11G $>A$ in the samples were found to be associated with mini-haplotype

Table 4. The types and frequencies of PAH-VNTR alleles among Iranian populations and other parts of the world

\begin{tabular}{|c|c|c|c|c|c|c|c|c|c|c|c|c|c|}
\hline \multirow{2}{*}{\multicolumn{2}{|c|}{ Population/geographic region }} & \multicolumn{12}{|c|}{ VNTR allele frequencies $(\%)$} \\
\hline & & 3 & 4 & 5 & 6 & 7 & 8 & 9 & 10 & 11 & 12 & 13 & 14 \\
\hline \multirow{7}{*}{ Iran } & Current study & 41.67 & & & & 11.80 & 31.95 & 11.80 & & & 2.78 & & \\
\hline & Iran (whole parts) (5) & 45 & & & 2.3 & 12.2 & 31.3 & 9.2 & & & & & \\
\hline & West Azerbaijan (23) & 50 & & & & & 49 & & & 1 & & & \\
\hline & Isfahan (21) & 45 & & 0.4 & 3 & 25 & 11 & 11 & 1 & 1.4 & & 2.4 & \\
\hline & Yazd (18) & 20 & & & & 20 & 33 & 13 & & 7 & 7 & & \\
\hline & Fars (20) & 44 & & & 4 & 13 & 26 & 9 & & & & 4 & \\
\hline & & 28 & & & & 12 & 44.8 & 11.2 & & & 4 & & \\
\hline
\end{tabular}

European Caucasians (6)

\begin{tabular}{|c|c|c|c|c|c|c|c|c|c|c|c|c|c|}
\hline \multirow{4}{*}{ Europe $^{\mathbb{}}$} & Adygei & 36 & & & 1 & 7 & 36 & 15 & & 4 & 1 & & \\
\hline & Karachay & 32 & & 1 & 1 & 9 & 37 & 15 & & 5 & & & \\
\hline & Kumyk & 41 & & 1 & & 7 & 37 & 1 & & 5 & & & \\
\hline & Nogay & 45 & & & 1 & 19 & 25 & 8 & & 2 & & & \\
\hline \multirow{5}{*}{$\begin{array}{l}\text { South } \\
\text { America }^{\mathbb{P}}\end{array}$} & Arara & 38 & & & & & 31 & 17 & 14 & & & & \\
\hline & Kayapo & 14 & & & & & 4 & 15 & 67 & & & & \\
\hline & Wayampi & 8 & & & & & 39 & 54 & & & & & \\
\hline & Wayana-Apalai & 34 & & & & & 1 & 24 & 32 & & & & \\
\hline & Yanomami & 4 & & & & & 1 & 17 & 69 & & & & \\
\hline \multirow{3}{*}{ Africa $^{\mathbb{R}}$} & Congo & & & & & 2 & 11 & 42 & 18 & 6 & 14 & & \\
\hline & Cameta' & 13 & & & & 10 & 28 & 33 & 9 & 2 & 12 & & 1 \\
\hline & Birongo & 16 & 3 & & & 5 & 31 & 25 & 5 & & 5 & 8 & \\
\hline
\end{tabular}

I; source: https://alfred.med.vale.edu/alfred/index.asp 
Table 5. PAH gene mutations and their linked mini-haplotypes in Iranian Kurdish population and some other populations around

\begin{tabular}{|c|c|c|c|c|}
\hline \multirow[t]{2}{*}{ Mutation } & \multicolumn{2}{|c|}{ Kermanshah province } & \multicolumn{2}{|c|}{ Other populations } \\
\hline & No. of alleles & Mini-haplotype & Population & Mini- haplotype \\
\hline IVS2+5G & 8 & $9 / 242$ & Germany & $9,8 / 242$ \\
\hline & & & Kuwait & $9 / \mathrm{ND}^{\mathrm{l}}$ \\
\hline $\begin{array}{l}\text { IVS9+5G } \\
>\mathrm{A}\end{array}$ & 6 & $8 / 238$ & $\begin{array}{l}\text { Turkey } \\
\text { Lebanon }\end{array}$ & $\begin{array}{l}\mathrm{ND}^{\mathrm{p}} \\
\mathrm{ND}^{\mathrm{p}}\end{array}$ \\
\hline IVS10- & 4 & $7 / 250$ & Australia & $7 / 242,246$ \\
\hline \multirow[t]{7}{*}{$11 \mathrm{G}>\mathrm{A}$} & & & Germany & $7 / 230$ \\
\hline & & & Germany & $7 / 242$ \\
\hline & & & $\mathrm{N}$ Ireland & $9 / 242$ \\
\hline & & & Spain & $7 / 250$ \\
\hline & & & Spain & $7 / 254$ \\
\hline & & & U. Kingdom & $7 / 240$ \\
\hline & & & U. Kingdom & $7 / 242,246$ \\
\hline \multirow[t]{5}{*}{ p.R261X } & 4 & $7 / 242$ & England & $8 / 242$ \\
\hline & & & Germany & $3 / 238,246$ \\
\hline & & & Norway & $7 / \mathrm{ND}^{\mathbb{R}}$ \\
\hline & & & Italy & $3 / \mathrm{ND}^{\mathrm{P}}$ \\
\hline & & & Fmr Soviet Union & $3 / \mathrm{ND}^{\mathrm{T}}$ \\
\hline p.K363> & 4 & $8 / 238$ & Kuwait & $8 / \mathrm{ND}^{\mathrm{R}}$ \\
\hline $\mathrm{Nfs}$ & & & Fmr Soviet Union & $8 / \mathrm{ND}^{\mathbb{}}$ \\
\hline \multirow[t]{4}{*}{ p.R243Q } & 2 & $9 / 250$ & Germany & $8 / 226,230$ \\
\hline & & & $\mathrm{N}$ Ireland & $9 / 234$ \\
\hline & & & Spain & $8 / 238$ \\
\hline & & & Spain & $8 / 242,246$ \\
\hline IVS7- & 2 & $8 / 242$ & ND & $\mathrm{ND}^{\mathrm{p}}$ \\
\hline $5 \mathrm{~T}>\mathrm{C}$ & & & & \\
\hline $\begin{array}{l}\text { IVS } 4+1 G \\
>C\end{array}$ & 2 & $7 / 242$ & ND & $\mathrm{ND}^{\mathrm{P}}$ \\
\hline
\end{tabular}

${ }^{\mathbb{N} N D}$; Not determined

$7 / 250$, which represents the ancestral background of this mutation.

Also, p.R261X, a nonsense mutation that leads to a premature step in translation, has been reported before in Iran and some countries such as Croatia, Italy, Brazil, Germany, Korea, Lithuania, and Portugal (19). This mutation has been found on mini-haplotypes 8/242, 3/238, 246, and $7 / 238$ in PKU patients with Caucasian (15), German (15), and Latvian (3) ethnicity (Table 5). The association of this mutation with mini-haplotype 7/242 in the present study shows that this linkage was not reported before. Moreover, p.R243Q, a common mutation in the Southeast Asian countries, was shown to be associated with $8 / 226$, $8 / 230,9 / 234$, and some other mini-haplotypes (15) (Table $5)$. In the present study, this mutation was linked to minihaplotype 9/250.

After PKU disease is confirmed in a newborn, identifying the 2 causing mutations is clinically useful to manage the disease. Mini-haplotypes are easier to obtain and are more informative for mutation analysis, including prenatal diagnosis, compared to conventional haplotypes. Determining mini-haplotypes proved very useful for the rapid identification of rare mutations. One of the drawbacks of mini-haplotype analysis is that it requires samples from the patient's parents, which may be a limitation in some cases. However, the knowledge of paternal or maternal inheritance is useful for carrier analyses in the extended family.

\section{Conclusion}

The distributions and frequencies of VNTR alleles in this Kurdish population have the most similarities to alleles previously described in European Caucasian families. Moreover, since the most common mutations in Kermanshah PKU chromosomes are rare and this was the first study on mini-haplotypes VNTR/STR among PKU patients in Iranian Kurdish PKU patients, and given that this study was the first of its kind, it was not possible to compare its results with that of other studies on Iranian and non-Iranian populations.

\section{Acknowledgments}

We thank the patients, their family members, and the healthy controls for their cooperation. This study was supported by a grant from Kermanshah University of Medical Sciences. The Vice Chancellor for Research at Kermanshah University of Medical Sciences has provided a grant to support this study.

\section{Conflict of Interests}

The authors declare that they have no competing interests.

\section{References}

1. Harding $\mathrm{CO}$, Blau N. Advances and challenges in phenylketonuria. J Inherit Metab Dis. 2010;33(6):645-8.

2. Hanley W. Phenylketonuria (PKU)-What Next? Mini-Review. J Genet Disor Genet Rep 2. 2013;2:2.

3. Pronina N, Lugovska R, editors. Association between minihaplotypes and mutations at the phenylalanine hydroxylase locus in Latvian phenylketonuria patients. Proceedings of the Latvian Academy of Sciences Section B Natural, Exact, and Applied Sciences; 2011.

4. Yubero D, Brandi N, Ormazabal A, Garcia-Cazorla À, Pérez-Dueñas $\mathrm{B}$, Campistol J, et al. Targeted Next Generation Sequencing in 
Patients with Inborn Errors of Metabolism. PloS One. 2016;11(5): e0156359.

5. Hosseini Mazinani S, Koochmeshgi J, Khazaee Koohpar Z, Hosein Pur Nobari N, Seifati S. Carrier detection of phenylketonuria in Iranian families by variable number tandem-repeat polymorphism analysis. East Mediterr Health J. 2008;14(6):1445-51.

6. Eisensmith RC, Goltsov AA, Woo SL. A simple, rapid, and highly informative PCR-based procedure for prenatal diagnosis and carrier screening of phenylketonuria. Prenat Diagn. 1994;14(12):1113-8.

7. Da Silva WA, Bortolini MC, Meyer D, Salzano FM, Elion J, Krishnamoorthy R, et al. Genetic diversity of two African and sixteen South American populations determined on the basis of six hypervariable loci. Am J Phys Anthropol. 1999;109(4):425-37.

8. Korshunova TY, Akhmetova V, Kutuev I, Khusainova R, Guseinov G, Khusnutdinova E. Analysis of the VNTR Polymorphism at the PAH and eNOS Genes and the CCR5 Gene Deletion in Populations of Northern Caucasus. Russ J Genet. 2004;40(3):321-5.

9. Aliakbari M, Khosravian F. Linguistic Capital in Iran: Using Official Language or Mother Tongue. Procedia Soc Behav Sci. 2014;98:190-9.

10. Andonian L, Rezaie S, Margaryan A, Farhud D, Mohammad K, Naieni $\mathrm{KH}$, et al. Iranian Azeri's Y-chromosomal diversity in the context of Turkish-speaking populations of the Middle East. Iran J Public Health. 2011;40(1):119.

11. Derenko M, Malyarchuk B, Bahmanimehr A, Denisova G, Perkova $\mathrm{M}$, Farjadian $\mathrm{S}$, et al. Complete mitochondrial DNA diversity in Iranians. PloS One. 2013;8(11):e80673.

12. Razipour M, Alavinejad E, Sajedi SZ, Talebi S, Entezam M, Mohajer N, et al. Genetic study of the PAH locus in the Iranian population: familial gene mutations and minihaplotypes. Metab Brain Dis. 2017;32(5):1685-91.

13. Liu N, Kong X, Zhao D, Wu Q, Li X, Guo H, et al. Prenatal diagnosis of Chinese families with phenylketonuria. Genet Mol Res. 2015;14(4):14615-28.

14. Kidd JR, Pakstis AJ, Zhao H, Lu R-B, Okonofua FE, Odunsi A, et al. Haplotypes and linkage disequilibrium at the phenylalanine hydroxylase locus, $\mathrm{PAH}$, in a global representation of populations. Am J Hum Genet. 2000;66(6):1882-99.

15. Scriver C, Prevost L, Hurtubise M, Konecki D, Dobrowolski S PAHdb Phenylalanine Hydroxylase Locus Knowledgebase. URL: http://www.pahdb mcgill ca, update. 2009;31.

16. Alibakhshi R, Moradi K, Mohebbi Z, Ghadiri K. Mutation analysis of PAH gene in patients with PKU in western Iran and its association with polymorphisms: identification of four novel mutations. Metab Brain Dis. 2014;29(1):131-8.

17. Moradi K, Alibakhshi R. High risk of birth defects with PKU in Mast-e Ali village, Kermanshah province. J Kermanshah Univ Med Sci. 2014;18(1):62-5.

18. Alibakhshi R, Moradi K, Biglari M, Shafieenia S. Spectrum of phenylalanine hydroxylase gene mutations in Hamadan and Lorestan Provinces of Iran and their associations with variable number of tandem repeat alleles. Iran J Med Sci. 2018;43(3):318.

19. Moradi K, Alibakhshi R, Ghadiri K, Khatami SR, Galehdari H. Molecular analysis of exons 6 and 7 of phenylalanine hydroxylase gene mutations in Phenylketonuria patients in Western Iran. Indian J Hum Genet. 2012;18(3):290.

20. Moradi K, Alibakhshi R, Alimadadi K. The frequency of the most common Mediterranean mutation in phenylketonuria patients in Kermanshah Province. Mol Genet Metab. 2014;19(1):58-66.

21. Kang MS, Choi SH, Koh IS. The effect of increasing control-to-case ratio on statistical power in a simulated case-control snp association study. Genomics Inform. 2009;7(3):148-51.

22. Parivar K, Seifati SM, Koochmeshgi J. Studying the level of informativity of VNTR marker on PAH gene for carrier detection of the patients with phenylketonuria in Yazd province, Iran. Med Sci J Islamic Azad Univ Tehran Med Branch. 2011;21(3):196-200.

23. Morteza B, Abdi RI, Hosseini JN, Rasoul Z, Ahad G. Frequency of the VNTR-Polymorphisms at the PAH Gene in the Iranian Azeri Turkish Patients with Phenylketonuria. Maedica. 2015;10(4):310.

24. Kamkar M, Saadat M, Saadat I, Haghighi G. Report of VNTR with 13 repeats linked to phenylalanine hydroxylase locus in unaffected members of two PKU families. Iran Biomed J. 2003;7(2):89-90.

25. Fazeli Z, Vallian S. An artifact band frequently associated with variable number of tandem repeat marker at phenylalanine hydroxylase gene: application in carrier detection and prenatal diagnosis of phenylketonuria. Mol Biol Rep. 2011;38(5):3395-9.
26. Fazeli Z, Vallian S. Estimation haplotype frequency of $\mathrm{BgIII} /$ EcoRI/VNTR markers at the PAH gene region in Iranian population. Int J Hum Genet. 2009;9(2):115-21.

27. Bagheri M, Isa Abdi R, Jazani Nh, Zarrin R, Ghazavi A. Association between PAH mutations and VNTR alleles in the West Azerbaijani PKU patients. Maedica. 2014;9(3):242.

28. Asadi S, Milani A, Nazirzadeh A, Manesh SG, Baegerpour N, Pourjafar R. Check the status of Haplotypes VNTR, MspI and PvuII (a) in the PAH gene in Tabriz population genotype data using Family Threesome. Bull Env Pharmacol Life Sci. 2015;4:101-6.

29. Rafiee L, Saadat I, Saadat M. Glutathione S-transferase genetic polymorphisms (GSTM1, GSTT1 and GSTO2) in three Iranian populations. Mol Biol Rep. 2010;37(1):155-8.

30. Fathzadeh M, Bigi MAB, Bazrgar M, Yavarian M, Tabatabaee HR, Akrami SM. Genetic counseling in southern Iran: consanguinity and reason for referral. J Genet Couns. 2008;17(5):472. 